\title{
Study of the shock motion in a hypersonic shock system/turbulent boundary layer interaction
}

\author{
C. B. Lee, S. Wang
}

\begin{abstract}
Wall pressure fluctuations and surface heat transfer signals have been measured in the hypersonic turbulent boundary layer over a number of compression-corner models. The distributions of the separation shock oscillation frequencies and periods have been calculated using a conditional sampling algorithm. In all cases the oscillation frequency distributions are of broad band, but the most probable frequencies are low. The VITA method is used for deducing large scale disturbances at the wall in the incoming boundary layer and the separated flow region. The results at present showed the existence of coherent structures in the two regions. The zero-cross frequencies of the large scale structures in the two regions are of the same order as that of the separation shock oscillation. The average amplitude of the large scale structures in the separated region is much higher than that in the incoming boundary layer. The length scale of the separation shock motion region is found to increase with the disturbance strength. The results show that the shock oscillation is of inherent nature in the shock wave/turbulent boundary layer interaction with separation. The shock oscillation is considered to be the consequence of the coherent structures in the separated region.
\end{abstract}

\section{1}

\section{Introduction}

From experimental evidence it is known that the shock system interacting with a turbulent boundary is unsteady and oscillatory (e.g. Settles et al. 1976; Dolling and Marphy 1982; Muck et al. 1985). The mechanism responsible for the shock oscillation is still not fully understood. Andreopoulos and

Received: 10 February 1993/ Accepted: 3 February 1995

\section{B. Lee}

Institute of Fluid Mechanics,

Beijing University of Aeronautics and Astronautics,

Beijing 100083, China

\section{S. Wang}

Institute of Mechanics, Chinese Academy of Sciences,

Beijing 100080, China

This work was supported by the Chinese National Science Foundation.

Thanks for Prof. Z. B. Lin and Miss X. Y. Feng for their helps. The authors wish to express thanks to Professor W. Merzkirch who has helped us to check the paper again and again.
Muck (1987) explained the shock oscillation with the bursts occurring in the upstream turbulent boundary layer, because they found that the frequencies of the shock oscillations and the burst phenomena are of the same order of magnitude. Tran and Bogdonoff (1987) argued differently. They processed their experimental data with the VITA technique and found different structures in the upstream boundary layer and in the intermittent region. They concluded that the influence of the upstream boundary layer on the shock oscillation is small and that the oscillations are dominated by the disturbances originating from the separated flow regime. According to Dolling and Smith (1989) the shock motion is driven by low frequency pressure fluctuations caused by the separated flow.

The present authors have performed a number of experimental studies in which the organized structures occurring in the upstream turbulent boundary layer and in the separated flow regime were analyzed (Lee and Wang 1989; Lee 1990; Wang and Lee 1991; Lee 1993; Lee and Lian 1993). One of the conclusions of these studies was that turbulent separation is a necessary condition for the shock oscillation.

Despite the great number of previous investigations many details of the unsteady shock phenomena are not well understood. The present paper aims at contributing to the further understanding of these processes. For this purpose measurements of the pressure fluctuations in the turbulent upstream boundary layer, the turbulent separated flow regime, and in the turbulent attached flow have been performed. Surface heat transfer measurements have been made as well. The experimental data were processed by using the conditional sampling algorithm (Andreopoulos 1986) and the VITA technique (Blackwelder and Haritoridis 1983). This analysis allows to conclude on some physical explanations of the mechanism responsible for the turbulent boundary layer/adverse pressure gradient interaction.

\section{2}

\section{Experimental procedure and data processing}

The gun tunnel, the compression-corner models, and the experimental techniques have been described in detail by Lee and Wang (1989). Therefore only a brief description is presented here. For all the experiments the stagnation conditions applied in the gun tunnel of the Institute of Mechanics were chosen as follows:

stagnation enthalpy: $h_{0}=4.44 \cdot 10^{4} \mathrm{~J} / \mathrm{kg}$,

stagnation pressure: $P_{0}=1.72 \cdot 10^{7} \mathrm{~Pa}$ 
Table 1. Dimensions of the ramp (see also Fig. 1)

\begin{tabular}{|c|c|c|c|c|}
\hline Inclination angle & $15^{\circ}$ & $30^{\circ}$ & $40^{\circ}$ & $90^{\circ}$ \\
\hline height $/ \mathrm{mm}$ & 15 & 309 & 35 & 18 \\
\hline width $/ \mathrm{mm}$ & 300 & 300 & 300 & 80 \\
\hline
\end{tabular}

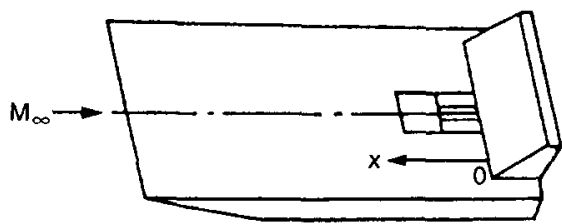

Fig. 1. Model and coordiante (one dimension)

The flow conditions upstream of the ramp are:

free stream Mach number: $M_{x}=7.8$

Reynolds number per meter: $R_{e}=3.5 \cdot 10^{7}$

boundary layer thickness: $\delta=6 \mathrm{~mm}$

ratio of wall and stagnation temperature: $T_{w} / T_{0}=0.39$

The model is a horizontal plate followed by an (inclined) two-dimensional ramp. Plate length and width are $500 \mathrm{~mm}$ and $300 \mathrm{~mm}$, respectively. The dimensions of the ramp are given in Table 1. The boundary layer on the horizontal plate is of zero pressure gradient, and it experiences a natural transition before reaching the ramp.

Pressure transducer (Kulite XCQ-80-25) of the solid resistance type (diameter $2 \mathrm{~mm}$, characteristic frequency $200 \mathrm{kHz}$ ) are arranged along the axial centerline with a distance of $3 \mathrm{~mm}$ between transducers. Surface heat transfer is measured by means of fast-response platinum-film resistance thermometers. The experimental data were stored in a 12 bit resp. 8 bit A/D multi-channel data-storage system that allows sampling at 20,50 and $500 \mathrm{kHz}$.

For the details of the VITA technique see Blackwelder and Haritoridis (1983). The technique has two degrees of freedom: the period of the short-time variance $\tau$, and the level of the threshold $K$. The physical interpretation of the peaks visible in the distribution of $\tau$ is still the subject of controversial discussions, but it is believed that the technique is useful for analyzing the present hypersonic flow phenomena (see also Spina and Smits 1987). The threshold is chosen as $K=0.8$ and $\tau$ in non-dimensional for as $\tau^{\star}=\tau \cdot U_{\infty} / \delta=7$.

3

\section{Experimental results and discussion}

\section{1}

\section{Surface pressure fluctuations in the intermittent region}

The upstream area of the corner can be divided into three regions: incoming turbulent boundary layer, intermittent region, and separation region as suggested by Muck et al. (1985). Photographs of the flow field of the shock

wave/turbulent boundary layer interaction are shown in Fig. 2.

The surface pressure fluctuations of the turbulent separated flow field were measured along the centerline upstream of the
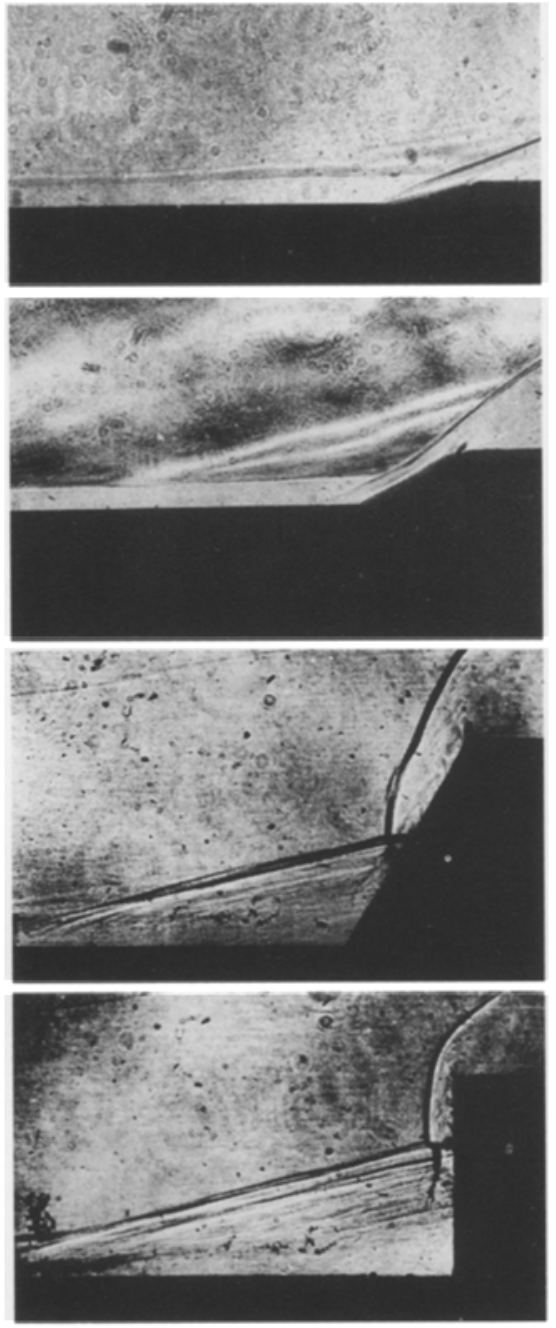

Fig. 2. Shadowgraphs of the flow fields upstream of the $15^{\circ}, 30^{\circ}, 60^{\circ}$, $90^{\circ}$ corners $\left(M_{\infty}=7.8\right) 15^{\circ}$ corner: attached flow; others: separated flow

$30^{\circ}, 40^{\circ}, 90^{\circ}$ compression corners; the results show a clear intermittency. The oscillations of the shock system induce surface pressure fluctuations as shown in Fig. 3(a). The length of the visible intermittent region can be acquired from the starting point to the end point of the intermittency. The incoming boundary layer is upstream of the starting point and the separation region is downstream of the end point.

We think that turbulent flow separation may be the necessary and sufficient condition for the shock system oscillations. We found it very important to study the turbulent separated flow field. Figure $3 \mathrm{~b}$, is the result of the surface heat transfer studies upstream of the $40^{\circ}$ corner. In the intermittent region ( $x \approx 98 \mathrm{~mm} \sim 116 \mathrm{~mm}$ ), the heat transfer is highly intermittent and jumps randomly back and forth between the incoming undisturbed boundary layer level and a higher disturbed level in the separation region.

The mean surface heat transfer and the pressure distributions along the centerline upstream of the $40^{\circ}$ corner are shown in Fig. 3c. In the beginning the mean heat transfer and the pressure both increase owing to an adverse pressure gradient. 

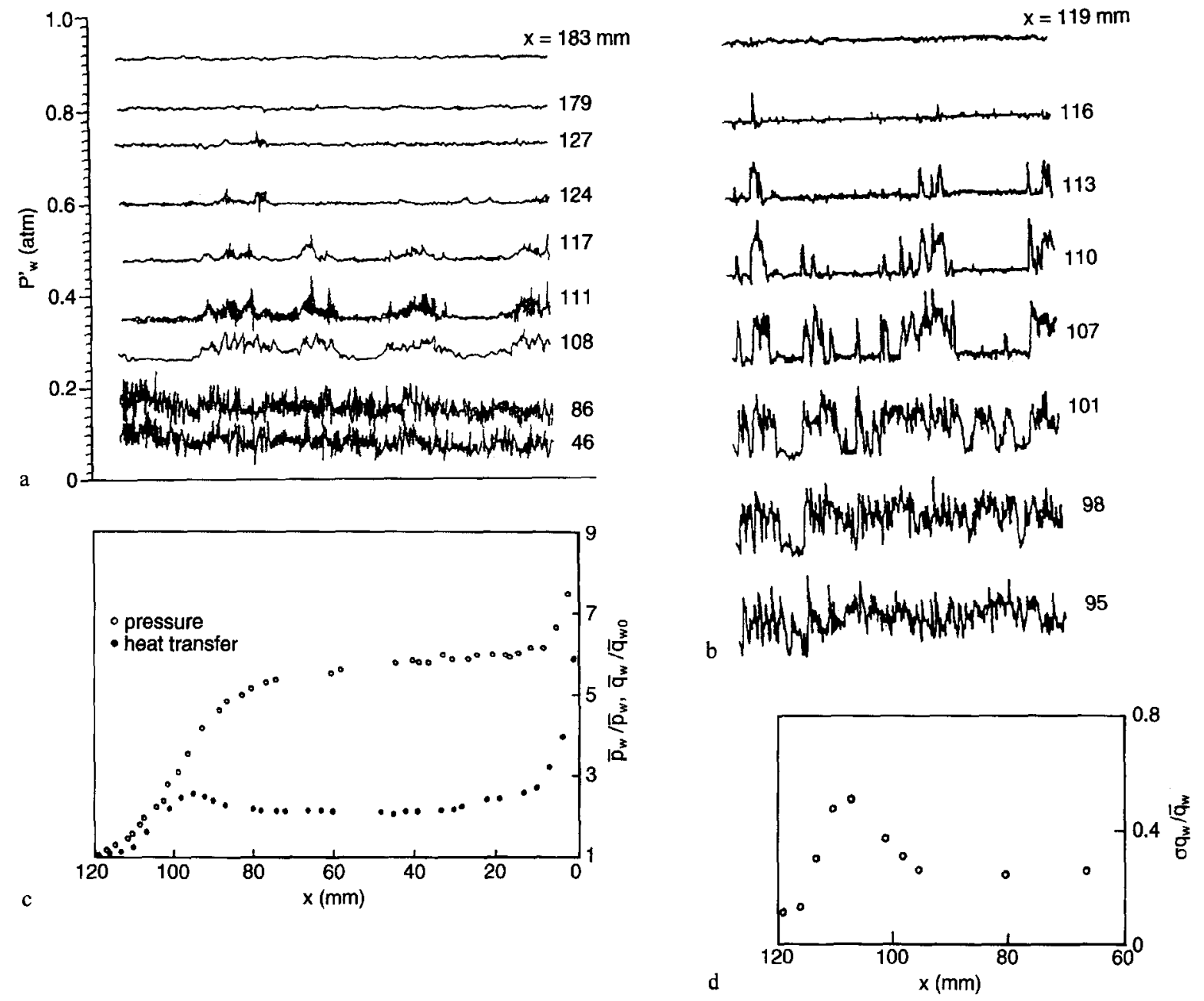

Fig. 3. a Surface pressure fluctuation upstream of the $90^{\circ}$ corner (sampling time $25 \mu$ s, total time $25.6 \mathrm{~ms}$ ); b Surface heat transfer upstream of the $40^{\circ}$ corner (sampling time $20 \mu \mathrm{s}$, total time $20.48 \mathrm{~ms}$, see also Fig. $3 \mathrm{c}$ and Fig. $3 \mathrm{~d}$; c Mean wall pressure and heat transfer distributions upstream of the $40^{\circ}$ corner; $\mathrm{d}$ Surface heat transfer standard deviation distribution $\left(40^{\circ}\right.$ corner $)$

Table 2. The zero-cross frequencies of the disturbances in the separated region and turbulent boundary layer for each measured point

\begin{tabular}{|c|c|c|c|c|c|c|c|c|c|c|c|c|c|}
\hline \multirow[b]{2}{*}{$X(\mathrm{~mm})$} & \multicolumn{4}{|c|}{ Boundary layer } & \multicolumn{3}{|c|}{$30^{\circ}$ Corner } & \multicolumn{6}{|c|}{$40^{\circ}$ Corner } \\
\hline & 124 & 127 & $124^{\mathrm{a}}$ & $127^{\mathrm{a}}$ & 1 & 4 & 7 & 6 & 19 & 33 & 36 & 39 & 42 \\
\hline Total time $(\mu \mathrm{s})$ & 1872 & 1924 & 4250 & 3835 & 1976 & 1612 & 1950 & 1872 & 1872 & 1898 & 1976 & 1916 & 1872 \\
\hline $\begin{array}{l}\text { Events (times) } \\
\mathrm{K}=0.8\end{array}$ & 8 & 7 & 16 & 14 & 8 & 7 & 8 & 9 & 7 & 9 & 8 & 9 & 7 \\
\hline $\mathrm{Fc}(\mathrm{KHz})$ & 4.27 & 3.64 & 3.76 & 3.65 & 4.05 & 4.34 & 4.10 & 4.84 & 3.74 & 4.7 & 4.05 & 4.55 & 3.74 \\
\hline
\end{tabular}

${ }^{\text {a }}$ sampling time is $2.5 \mu \mathrm{s}$ for each measured point and others $1.0 \mu \mathrm{s}$

Figure $3 \mathrm{~d}$ gives the distribution of the surface heat transfer standard deviation normalized by the local mean heat transfer upstream of the corner.

\section{2}

\section{Relationship between flow structures upstream and downstream of the shock system}

In the intermittent region, the pressure fluctuations jump repeatingly from the value in the incoming boundary layer to that in the separated region. In order to realize the process of the shock system oscillation, the conditional sampling technique must be used. It was found in our previous works (Lee and Wang 1989, Lee 1990) that the frequencies of the shock system oscillation are in a broad band. The maximum zero-cross frequency $F_{\mathrm{c}}$ of the shock system oscillation is about $4.0 \mathrm{KHz}$ for all three corners.

The surface pressure fluctuations of the incoming turbulent boundary layer, a random structure of Gaussian distribution, are different from that of the shock/boundary layer interaction region. The frequencies of the disturbances in the separation 
Table 3. Amplitudes of the disturbances in the downstream and upstream parts of shock system

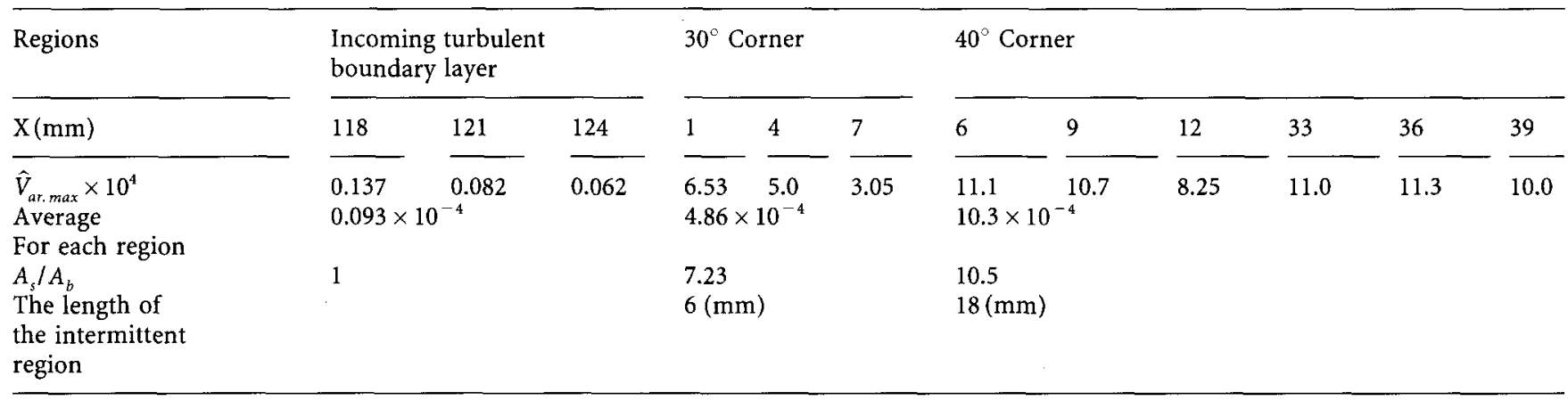

$\hat{V}_{a r_{\text {max }}}$ : The average of all the $\hat{V}_{a r, \text { max }}$, which is the maximum value of $\hat{V}_{a r}$, in a period of disturbance. $A_{s}, A_{b}$ are the average amplitudes of the disturbances in the separated flow region and the incoming turbulent boundary layer. $\hat{V}_{a r}$ is VITA measure function.
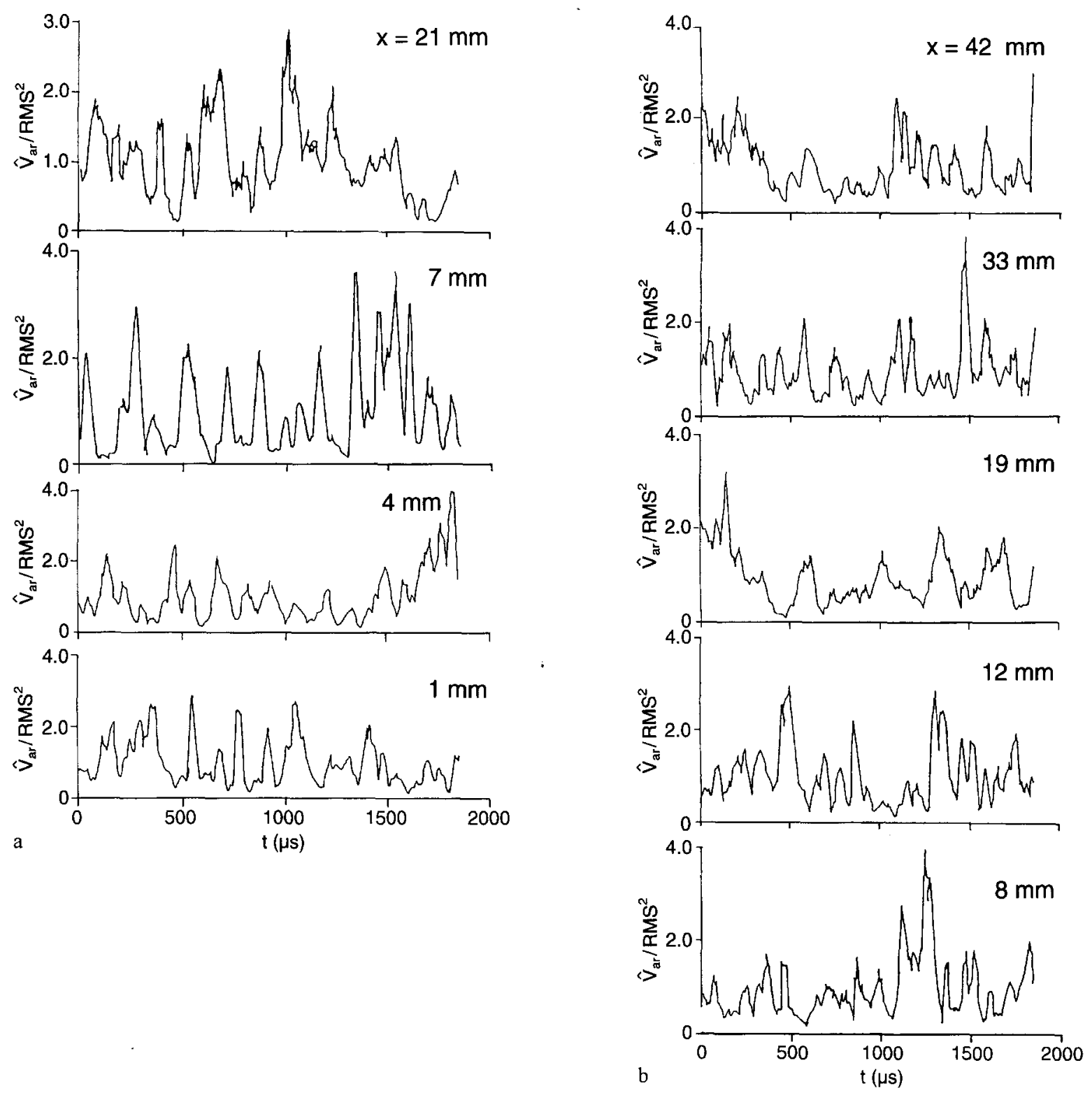

Fig. 4. a The VITA pressure events $x=21 \mathrm{~mm}$ in boundary layer, others in separated region; RMS is the root of mean square of the fluctuations $\left(30^{\circ}\right.$ corner); $\mathbf{b}$ The VITA pressure events in separated region $\left(40^{\circ}\right.$ corner) (sampling time $\left.1 \mu \mathrm{s}\right)$ 
region and in the incoming turbulent boundary layer are obtained by using the VITA technique, which shows that there exist low frequency disturbances not only in the incoming turbulent boundary layer but also in the separated region. The frequencies of the disturbances in the incoming turbulent boundary layer and in the separated region are shown in Table 2. From Table 2, the frequencies of disturbances in the boundary layer and separated region are of the same order of magnitude.

Spina and Smits (1987) have proved that there exists a correlation between the pressure fluctuations and the massflux. Owen and Horstman (1972) found that the transport of disturbances in a hypersonic turbulent boundary layer is similar to that in an incoming turbulent boundary layer at low subsonic flow. Simpson et al. (1981) visualized the coherent structures in the separation region at low speeds. Lian (1990) visualized the coherent structures in the turbulent boundary layer with adverse pressure gradient. All their results confirm that the turbulent separated layer is naturally unstable. Despite the shortage of the detailed information in the study of the hypersonic separated layer, the characteristics of disturbances in the separated layer may be considered to be the same as that at low subsonic flow.

The amplitude of the large scale structures can easily be determined by the VITA technique. The average value of the amplitudes in the separation region is about $7 \sim 10$ times larger than that in the incoming turbulent boundary layer (see Table 3). This shows that the amplitudes of the disturbances in the incoming turbulent boundary layer are low. Although the large structures in the turbulent boundary layer have a low frequency, the amplitude is too small to induce the shock oscillation at high amplitudes and low frequencies. The results also reveal that the length of the intermittent region and the amplitudes of the disturbances in the separated region are directly proportional to the corner angles.

Figure 4a shows the large scale structures of the pressure fluctuations in the separated region and the region of the incoming turbulent boundary layer upstream of the $30^{\circ}$ corner.

Figure $4 \mathrm{~b}$ gives the same structures in the separated region upstream of the $40^{\circ}$ corner.

All the results indicate that the shock system oscillation is not caused by the disturbances in the incoming turbulent boundary layer but by the disturbances in the separation region.

Figure 5 gives the space/time correlation of the measurements in the separation region upstream of the $40^{\circ}$ corner. All the points measured are in the separation region. The convection velocity $U_{c}$ of the large scale structures is obtained such that $U_{c}=0.55 U_{\infty}$.

From Fig. 6 it follows that there exists a correlation between the large structures in the separated region and the zero-pressure gradient turbulent boundary layer. The reason for the small correlation value was that the distance between the two measured points was chosen relatively long. Two evidences, the correlation result and the same order frequencies of the large scales in the boundary layer and separated flow region, show that the large scale structures in the incoming turbulent boundary layer may affect the formation of similar structures in the separated flow region.

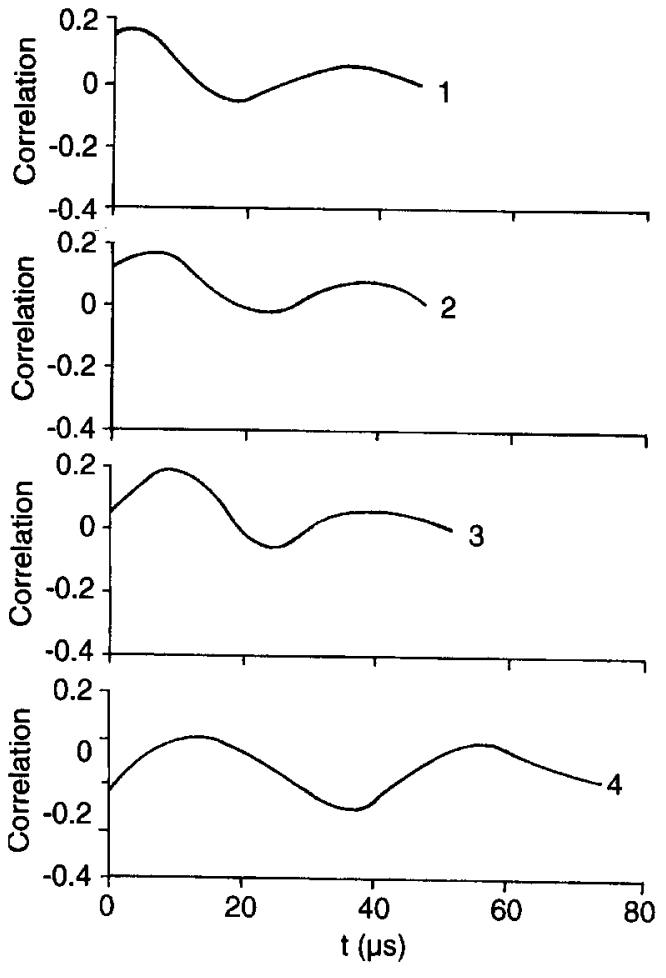

Fig. 5. The space-time correlation of pressure in the separated region $1 \cdots x=42,39 ; 2 \cdots x=42,36 ; 3 \cdots x=42,33 ; 4 \cdots x=42,13$

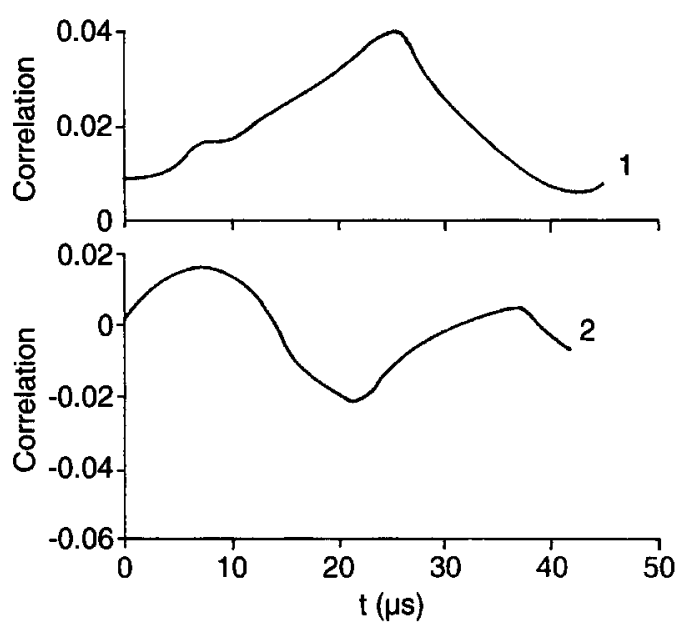

Fig. 6. The space-time correlation of pressure between separated region and boundary layer. $1 \cdots x=45 \mathrm{~mm}, 15 \mathrm{~mm} ; 2 \cdots x=4 \mathrm{~mm}$, $27 \mathrm{~mm}$

By using the convection velocity and the time scale of the large scale structures (see Fig. 5), a spatial scale of the structures can be obtained with the Taylor vortex scale formula: $L=U_{c} \star t$, which shows that the scale is of the same order of magnitude as the thickness of the separated layer. Here the time scale $t$ of the large scale can be acquired from Fig. 4a. The structures exhibit three characteristics: high amplitude, low frequency and an equivalent spatial scale of the separated layer. In other words, the structures acquired by processing the pressure fluctuations are coherent structures in the separated layer. 
Figure 7 is the result obtained by Image Processing Techniques in the IPT center of BUAA. According to Hayashi et al. (1989), the image resolution is 8 bit $* 8$ bit. Fig. 8 is the result obtained by applying VITA to the data of Fig. 7. The technique has a high accuracy because we only investigate the low frequency characteristics of the disturbances. These studies show that there exist coherent structures in the incoming boundary layer and in the separation region as obtained from the heat transfer fluctuations. The frequencies of the coherent structures in the two regions and those of the shock oscillation are of the same order of magnitude.
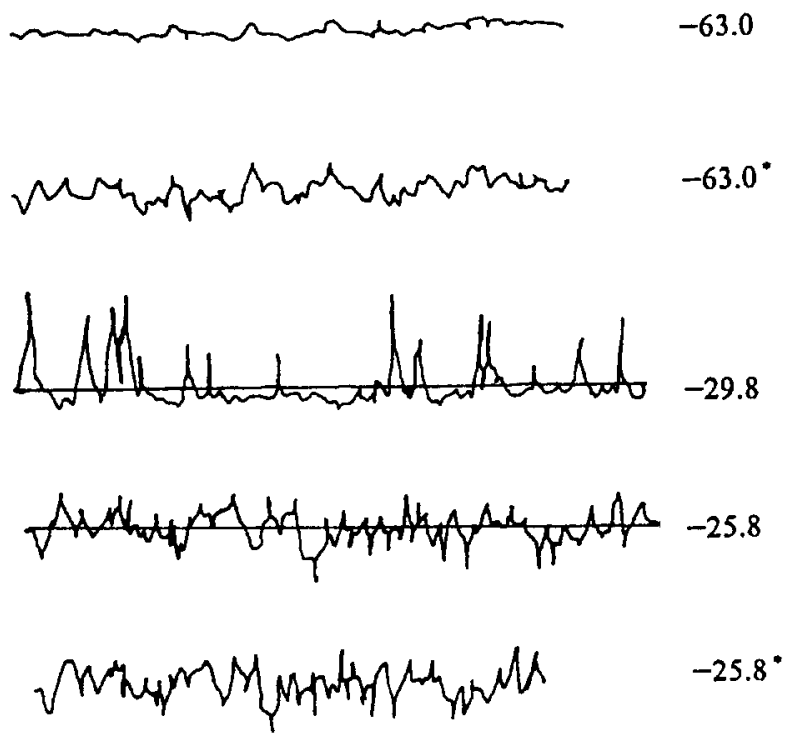

Fig. 7. Signals of the heat flux.

-63.0 in boundary layer (B.L.)

-29.8 in the intermittent region (I.R.)

-25.8 in the separated region (S.R.)

*By using image processing technique (I.P.T.)
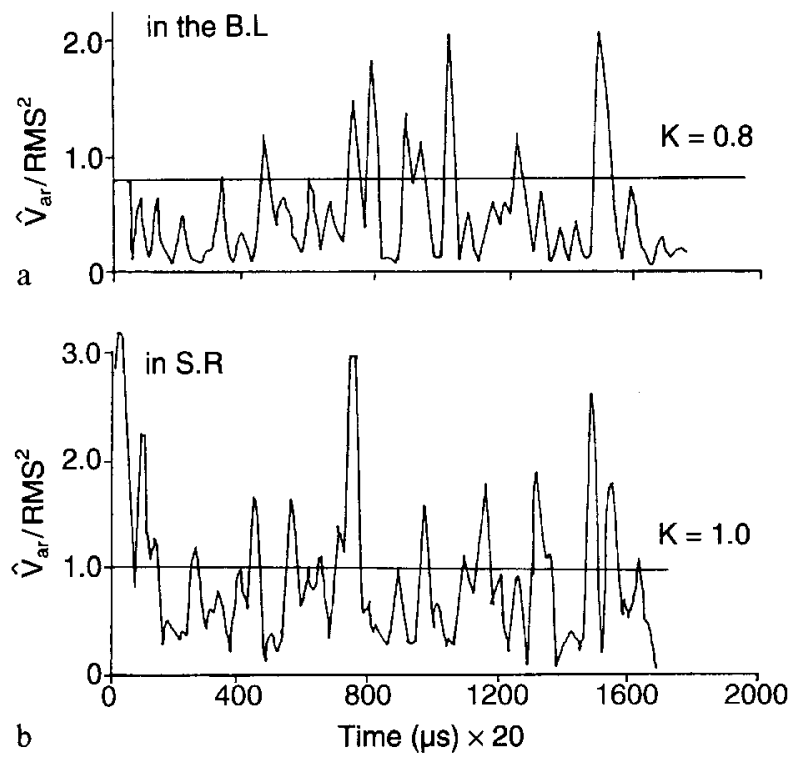

Fig. 8a, b. The VITA events by applying VITA to the data of Fig. 7

\section{4}

\section{Discussion}

For the investigated different Mach numbers and different boundary layer conditions, all the results obtained from both heat transfer and pressure fluctuations show that the shock wave oscillation is driven by the coherent structures in the separated layer.

The coherent structures in the separated region are controlled by two factors: the incoming turbulent boundary layer an the adverse pressure gradient. The instability is caused by the turbulent boundary layer. The adverse pressure gradient plays an important role in the coherent structures in the separated region.

The shock system oscillation may be classified into 4 types, namely high frequency and high amplitude, high frequency and low amplitude, low frequency and high amplitude and low amplitude and low frequency. Because of the unknown physical reasons and the limitation of the available measurement techniques, we only discuss the shock system oscillation at low frequency and high amplitude.

In general, the shock system oscillation can be considered as being driven by the coherent structures in the separated region; the coherent structures in the separated region are controlled by the equivalent structures in the incoming turbulent boundary layer and by the adverse pressure gradient.

\section{5}

\section{Conclusion}

(1) The mechanism of the shock system oscillation is governed by the coherent structures in the separated region. The adverse pressure gradient amplifies the structures and also affects the frequencies of the structures. The frequency of the structures is affected by the instability of the incoming turbulent boundary layer.

(2) All the factors which affect the bursting of the turbulent boundary layer control the structures in the separated region. As a result of the incoming turbulent boundary layer and the adverse pressure gradient interaction, the shock system oscillation is a typical result of the pressure wave and the turbulent wave interaction. In fact, the physical process of the shock system oscillation is a phenomenon of turbulence.

\section{References}

Andreopoulos J; Muck KC (1987) Some new aspects of the shock wave boundary layer interaction in compression ramp flows. J Fluid Mech 180: 405-428

Blackwelder RE; Haritoridis JH (1983) Scaling of the bursting frequency in turbulent boundary layer. J Fluid Mech 132: 87-101

Dolling DS; Murphy M (1982) Wall pressure in a supersonic separated compression ramp flow field. AIAA Paper 82-0986

Dolling DS; Or CT (1983) Unsteadiness of the shock wave structure in attached and separated compression ramp flow field. AIAA Paper 83-1715

Dolling DS; Smith DR (1989) Separation shock dynamics in Mach 5 turbulent interaction induced by cylinders. AIAA J 27: 1698-1706 Lee CB (1990) The mechanism of the shock system oscillation in the shock system turbulent boundary layer interaction. Master Thesis of the Inst. of Mech., Chinese Academia Sinica

Lee CB (1993) Some problems in the shock system/turbulent boundary layer interaction. Proc. of IUTAM Symp. (S.P. Lin Eds.), Potsdam, NY, 213-231. Springer 
Lee CB; Lian QX (1993) Some new problems in the shock/turbulent boundary layer interaction. In: Proc. of the 4th Annual Symp. of Beijing, 1-3

Lee CB; Wang Shifen (1989) Preliminary investigation of the mechanism of the turbulent separated shock wave oscillation, Rep. of the 4th National Symp. on Separated Flow and Wave Motion, Ximen, China

Lian QX (1990) A visual study of the coherent structure of the turbulent boundary layer in flow with adverse pressure gradient. I Fluid Mech 215: 101-124

Muck KC; Dussauge JP; Bogdonoff SM (1985) Structure of the wall pressure fluctuations in shock-induced separated turbulent flow. AIAA Paper 85-0179

Hayashi M; Aso S; Tan A (1989) Fluctuation of heat transfer in shock wave/turbulent boundary-layer interaction. AIAA J 27: 399-404

Owen FK; Horstman CC (1972) On the structure of hypersonic turbulent boundary layers. J Fluid Mech 53: 611-636
Settles GS; Fitzpatrick TT; Bogdonoff SM (1979) Detailed study of attached and separated compression corner flowfields in high Reynolds number supersonic flow. AIAA J 17: 579-585

Simpson RL; Chew YT; Shivaprasad BG (1981) The structure of a separating turbulent boundary layer. J Fluid Mech 113: 53-73

Spina EF; Smits AJ (1987) Organized structures in a compressible turbulent boundary layer. J Fluid Mech 182: 85-109

Smits AJ; Muck KC (1987) Experimental study of three shock wave/ turbulent boundary layer interactions. J Fluid Mech 182: 291-314

Tran TT; Bogdonoff SM (1987) An experimental investigation of unsteadiness in swept shock wave/turbulent boundary layer interactions. AIAA Paper 87-552

Wang Shifen; Lee CB (1991) An experimental study of the unsteady natural of the shock-induced hypersonic turbulent separated flow. Proc. of the International Conference on Experimental Fluid Mech., Chengdu, 101-106 